\title{
Sexual and gender based violence in Africa: Key issues for
} programming

Population Council

Follow this and additional works at: https://knowledgecommons.popcouncil.org/departments_sbsr-rh

Part of the Demography, Population, and Ecology Commons, Domestic and Intimate Partner Violence Commons, Family, Life Course, and Society Commons, and the International Public Health Commons How does access to this work benefit you? Let us know!

\section{Recommended Citation}

"Sexual and gender based violence in Africa: Key issues for programming." Nairobi: Population Council, 2008. 


\section{SEXUAL AND GENDER BASED VIOLENCE IN AFRICA:}

KEY ISSUES FOR PROGRAMMING

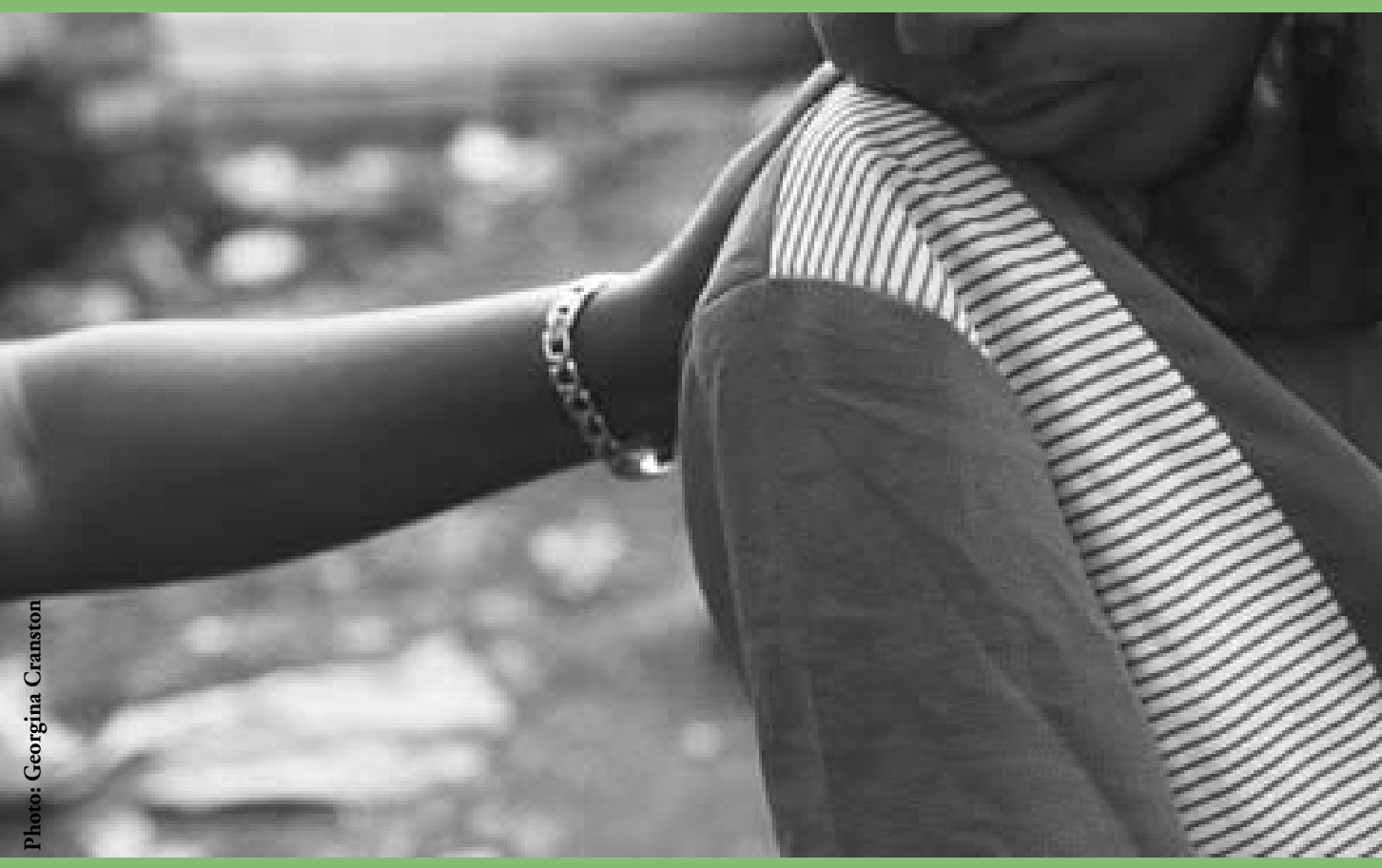


Sexual and Gender Based Violence (SGBV), in its various forms, is endemic in communities around the world, cutting across class, race, age, religion and national boundaries. Exposure to gender-based violence and sexual coercion significantly increases girls', and women's risk of early sexual debut, experiencing forced sex, engaging in transactional sex, and unprotected sex. The impact of sexual and gender-based violence resonates in all areas of health and social programming: survivors of sexual violence experience increased rates of morbidity and mortality, and violence has been shown to exacerbate HIV transmission, among other health conditions [1]. While women are the most visible survivors of sexual violence, they are far from being the only ones who suffer from the consequences: children of both sexes constitute the majority of abuse survivors reporting for medical and police services, and adult men and the handicapped are groups who are often neglected in research and interventions.

This brochure summarises the key points from a literature review on SGBV designed to inform partners across Africa. The review is a resource for developing a comprehensive model of care, support and prevention that partner countries can adapt, as a whole or in part. It is structured around a set of components, outlined in the box on page 1 , that are collectively designed to meet the full range of survivors' medical, psychological and justice needs in an integrated manner, while contributing to community level prevention efforts.

\section{SGBV DEFINITIONS AND CLASSIFICATIONS}

The term sexual and gender based violence (SGBV), in its widest sense, refers to the physical, emotional or sexual abuse of a survivor.

Sexual violence is defined as "any sexual act, attempt to obtain a sexual act, unwanted sexual comments or advances, or acts to traffic women's sexuality, using coercion, threats of harm or physical force, by any person regardless of relationship to the survivor, in any setting, including but not limited to home and work" [2]. The scope of the definition is here expanded to include the forced sex, sexual coercion and rape of adult and adolescent men and women, and child sexual abuse [25]. The definition also includes:

- The use of physical violence or psychological pressure to compel a person to participate in a sexual act against their will, whether or not the sexual act is consummated.

- A sexual act (whether attempted or consummated) involving a person who is incapable of understanding the nature or significance of the act, or of refusing, or of indicating his or her refusal to participate in the act.

- Abusive sexual contact.

Gender-based violence* is "physical, mental, or social abuse that is directed against a person because of his or her gender or gender role in a society or culture. In these cases, a person has no choice to refuse or pursue other options without severe social, physical, or psychological consequence"[1].

*The term gender-based violence is widely used as a synonym for violence against women, in order to highlight the gender inequality in which much violence is rooted [1]. 


\section{The African Regional SGBV Network}

The Population Council is supporting a multi-sectoral and multi-country network of partners dedicated to strengthening SGBV services. Initiated in 2006, the network aims to promote a comprehensive approach to preventing and managing SGBV throughout the region.

Recognizing the need for clear guidelines for programmes addressing SGBV and a holistic response to SGBV in Africa, the network is guided by a broad comprehensive framework (see box). Implementing partners aim to:

- develop feasible, effective and efficient models for a comprehensive response to the needs of SGBV survivors;

- strengthen services that address the health, psychological and criminal justice consequences of violence; and

- reduce the determinants of violent behaviour within communities.

\section{Framework for a comprehensive model of care, support and} prevention of SGBV

1. Medical management of sexual violence at point of first contact with the survivors.

2. Psychological counselling of rape survivors.

3. Sensitive approaches to managing child survivors of sexual violence (of both sexes), and to encouraging and enabling presentation by male survivors.

4. Collection of forensic evidence (at health facility during medical management and/or at police station) and creation of a chain of evidence that can be used during a prosecution.

5. Strong links between police and health facility to enable incidents to be referred in either direction so that, if desired, a prosecution can be initiated. Ensure prosecutions initiated by the police are sustained through the judiciary.

6. New or strengthened community-based prevention strategies that are relevant and appropriate for the local context and that are directly linked to the nearest medical/police structures.

7. Physical (and psychological/emotional) violence between domestic or intimate partners addressed through:

a. Messages communicated during the prevention strategies;

b. Screening for signs and symptoms of such violence during routine health consultations. 


\section{SGBV in Africa}

\section{Causes \& risk factors}

Social, economic, and gender issues are increasingly recognized as significant factors in Africa that underlie the HIV epidemic, keep maternal mortality and fertility rates high, and increase the likelihood that sex will not be safe, voluntary, or pleasurable.

Certain community and societal-level risk factors are associated with higher or more severe rates of sexual and gender-based violence [2]:

- Traditional gender norms that support male superiority and entitlement

- Social norms that tolerate or justify violence against women

- Weak community sanctions against perpetrators

- Poverty

- High levels of crime and conflict in society more generally.

Research on violence against women shows an increased risk of current physical or sexual violence among women of a younger age (especially those aged 15 to 19) [2,26, 27] and with lower levels of education [26]. Women who are separated or divorced (or, to a lesser degree, cohabiting) report a higher lifetime prevalence of all forms of violence [26]. Alcohol or drug consumption, and previous experience of sexual abuse, also correlate with sexual violence in adulthood [2].

Research into individual-level risk factors indicates violence is a learned behaviour: for instance, boys who witness or experience violence as children are more likely to use violence against women as adults, and a history of sexual abuse distorts perceptions about sexual violence and the risk of HIV infection [1, 3].

\section{Consequences}

Violence, and the fear of violence, severely limits survivors' contribution to social and economic development, thereby hindering achievement of the Millennium Development Goals and other national and international development goals.

Epidemiological evidence shows that violence is a major cause of ill health among women and girls, as seen through death and disabilities due to injuries, and through increased vulnerability to a range of physical and mental health problems $[2,4]$.

Both men and women can be survivors or perpetrators of violence. It is important to recognise, however, that although male against female violence is more common, a not insignificant proportion of males, and especially boys, suffer all four types of violence outlined below. 


\section{Health consequences of intimate partner violence and sexual violence}

\begin{tabular}{|c|c|c|c|}
\hline \multicolumn{3}{|l|}{ Non fatal outcomes } & Fatal outcomes \\
\hline $\begin{array}{l}\text { Physical injuries and } \\
\text { chronic conditions }\end{array}$ & $\begin{array}{l}\text { Sexual and reproductive } \\
\text { sequelae }\end{array}$ & $\begin{array}{l}\text { Psychological and behavioural } \\
\text { outcomes }\end{array}$ & \multirow{3}{*}{ Femicide } \\
\hline Fractures & Gynecological disorders & Depression and anxiety & \\
\hline Abdominal/thoracic injuries & Pelvic Inflammatory disease & Eating and sleep disorders & \\
\hline Chronic pain syndromes & $\begin{array}{l}\text { Sexually-transmitted } \\
\text { infections, including HIV }\end{array}$ & Drug and alcohol abuse & \multirow[t]{2}{*}{ Suicide } \\
\hline Fibromyalgia & Unwanted Pregnancy & Phobias and panel disorder & \\
\hline Permanent disability & Pregnancy complications & Poor self-esteem & \multirow{4}{*}{$\begin{array}{l}\text { AIDS-related } \\
\text { mortality }\end{array}$} \\
\hline Gastrointestinal disorders & Miscarriage / low birth weight & Post-traumatic stress disorder & \\
\hline Irritable bowel syndrome & Sexual dysfunction & Post-traumatic stress disorder & \\
\hline Lacerations and abrasions & Unsafe abortion & Self harm & \\
\hline Ocular damage & & $\begin{array}{l}\text { Unsafe sexual behaviour: } \\
\text { - high-risk views on sexual } \\
\text { violence \& HIV infection } \\
\text { - less likely to use condoms } \\
\text { \& contraceptives }\end{array}$ & $\begin{array}{l}\text { Maternal } \\
\text { mortality }\end{array}$ \\
\hline
\end{tabular}

Sources: Adapted from Heise and Garcia Moreno, 2002; and Heise, Ellsberg and Gottemoeller, 1999 [5].

\section{Prevalence of SGBV in Africa}

Gender-based violence and forced sex are highly prevalent in sub-Saharan Africa:

- In Zambia, DHS data indicate that $27 \%$ of ever-married women reported being beaten by their spouse/partner in the past year; this rate reaches $33 \%$ of $15-19$ year-olds and 35 percent of $20-24$ year-olds. $13 \%$ of $15-19$ year olds were sexually coerced in the past 12 months [28].

- In South Africa, $7 \%$ of 15-19 year-olds had been assaulted in the past 12 months by a current or ex-partner; and $10 \%$ of $15-19$ year-olds were forced or persuaded to have sex against their will [30]

- In Kenya, $43 \%$ of $15-49$ year old women reported having experienced some form of gender-based violence in their lifetime, with $29 \%$ reporting an experience in the previous year; $16 \%$ of women reported having ever been sexually abused, and for $13 \%$, this had happened in the last year [6].

- In rural Ethiopia, 49\% of ever-partnered women have ever experienced physical violence by an intimate partner, rising to $59 \%$ ever experiencing sexual violence [26].

- In rural Tanzania, $47 \%$ of ever-partnered women have ever experienced physical violence by an intimate partner, while $31 \%$ have ever experienced sexual violence [26]. 


\section{Key components of medical management of SGBV}

Comprehensive post-rape care aims to reduce the physical and psychological consequences of sexual violence $[10,11,12]$. An integrated care package includes:

Treatment of injuries,

clinical evaluation and

forensic examination

Pregnancy testing and

emergency contraception (EC)
- Survivors of sexual abuse may have physical injuries that require immediate attention. Life-threatening injuries take precedence over other components of medical management $[12,16]$.

- The components of the clinical evaluation - forensic examination, specimen collection, analysis and documentation - act as a vital link between health care and the judicial system [13].

- EC should be available to all female survivors of rape who are of reproductive age, who are: not pregnant, not consistently using a reliable form of contraception, and who show signs of secondary sexual development [13, 14]. A pregnancy test is not required prior to administering EC. A pregnancy test is desirable, however, to determine eligibility. It is important to reassure clients that the EC pills will cause no harm to an existing foetus or to the course of the pregnancy [14].

- In environments where dedicated EC drugs are not available, health providers can offer combinations of oral contraceptive pills.

- EC can be administered within 120 hours of the assault, but is most effective when given as early as possible, so its provision is a priority, along with HIV prophylaxis [12]. An antiemetic can be offered alongside EC to reduce the chance of vomiting.

- PEP involves the administration, within 72 hours of sexual HIV diagnostic testing and counselling (DTC) and Post Exposure Prophylaxis (PEP) penetration, of one or a combination of anti-retroviral drugs (ARVs) to HIV negative persons, to be continued for 28 days after penetration [12,16]. A stat dose of PEP can reduce the time interval to first dose [37].

- Because of the elevated risk of HIV transmission in high prevalence settings in many parts of Africa, it is recommended that PEP should be available at the first point of entry to a health facility in high prevalence settings [13]. 
HIV DTC and PEP

(ctd.)

Prophylaxis of sexually transmitted infections

(STIs)

Trauma counselling
- HIV DTC is recommended to precede the administration of PEP [12]. Most patients present to health facilities out of hours, therefore VCT should be made available 24 hours a day [37].

- In rural areas, few patients are able to return to hospital after the initial presentation. Therefore, wherever possible, all diagnostic tests and treatment should be provided on the first visit. For those who are HIV negative, a full 28day course of PEP should be dispensed on the first visit. Same-day provision of anti-emetics and medication counselling are important for encouraging adherence [37].

- When feasible, WHO recommends that patients be tested for chlamydia, gonorrhoea, trichomoniasis, syphilis and hepatitis $B$, although this may vary according to local environments and national protocols [16].

- The incubation periods of different STIs vary, and follow-up tests are advisable [16]. Treatment may relieve a source of stress, but the decision about whether to receive prophylactic treatment or wait for results of STI tests should be made by the woman [28].

- When STI testing is not feasible, the Kenyan $\mathrm{MoH}$ recommends that post-exposure prophylaxis of STIs should be commenced at an early stage of treatment (within 24 hours) $[12,38]$.

- Emotional consequences of sexual assault as expressed in the 'rape trauma syndrome' are often longer lasting and more difficult to diagnose and deal with than physical symptoms. Trauma counselling is a crucial component of all services (see section $\mathrm{V}$ ).

\section{Male survivors of sexual violence}

Men most commonly experience sexual violence in the form of: receptive anal intercourse; forced masturbation of the perpetrator; receptive oral sex; forced masturbation of the victim [16]. Due to stigma and prejudice regarding male sexuality, men are less likely to seek medical care, legal or psychosocial support [2, 36].

Male survivors of sexual violence require the same physical examination and medical interventions as women, although the genital examination requires a specific approach [16]. Counsellors may also need to reassure them about perceived challenges to their sexuality or masculinity. 


\section{Children and Sexual Violence}

Children are especially vulnerable to sexual violence by nature of their relatively weak social position, economic dependence and lack of political protection:

- The World Health Organization estimated in 2001 that 40 million children are annually subjected to physical or sexual abuse [7].

- A strong association exists between early sexual initiation and coercion, particularly among girls. Population-based surveys in South Africa recorded $28 \%$ of girls reporting forced sexual initiation [2]. In provincial Tanzania and urban Namibia, 43\% and $33 \%$ respectively of women reporting first sex before the age of 15 years described that experience as forced [26].

- Myths that sex with young virgins can cleanse the perpetrator of sexual assault of the HIV virus have contributed to the rising phenomenon of child rape in Africa [8].

\section{Medical Management of Children}

While the medical management of children is broadly similar to that of adults, there are certain crucial differences in care and the administration of drug regimens.

- Children manifesting severe physical injuries may be examined under anaesthesia, while children with less physical trauma can be treated and then referred [12].

- Routine administration of STI prophylaxis is recommended in high prevalence settings, although the dosage levels are child-specific. A follow-up visit to check for emerging STI cultures is recommended in cases where sexual abuse has recently occurred [16].

- PEP regimens for children can consist of syrups or tablets, or a combination of both $[12,16]$. Children require lower dosage than adults, and with tablets, weight bands can be used to determine paediatric doses.

- Paediatric PEP protocols state that HIV testing need not precede PEP provision, to reduce delays [17].

- Many countries have laws requiring mandatory reporting of cases of child abuse to the local authorities or police, and health care workers should be aware of the obligations in their own country. 


\section{Legal and judicial responces to sexual violence}

\section{Forensic evidence}

Forensic evidence is usually needed to confirm the occurrence of sexual assault and to prove or disprove a link between the alleged perpetrator and the assault, and thus to secure prosecution and sentencing $[12,16]$. An efficient system requires a "chain of evidence" that allows forensic evidence collected from the health facility to be sent to a forensic laboratory for analysis and then on to the police for further action.

The health provider conducting the medical assessment should ideally be the person providing the forensic service. The following principles for examination and specimen collection are recommended [16, 13]:

- Full occurrence and medical history, documented on a government-approved form, which is admissible as evidence in court.

- Careful collection, avoiding contamination, and appropriate handling, storage and transport of specimens from the point of collection to the forensic laboratory (to include torn or soiled clothing, any depositions on the survivor's body, and a vaginal, anal or oral swab).

- Timely collection - the value of the evidentiary material decreases significantly 72 hours after the assault.

- Accurate labelling and efficient documentation of all collection and handling procedures.

- Documentation of the chain of custody of specimens.

\section{Inter-sectoral collaboration and referral linkages}

Inter-sectoral collaboration is a key determinant of the quality of comprehensive services. The 72-hour 'window of opportunity' for forensic examination and medical management signifies the importance of quick and efficient referrals between police, health, and judicial institutions. Effective referral mechanisms need to be established simultaneously with strengthening the component services.

The law enforcement and justice sectors play key roles in addressing the needs of SGBV survivors. Both female and male survivors need access to appropriate legal advice and resources, as well as counselling and support for their medical and psychological needs. This can be achieved by:

- building the capacity of local para-legal and community organizations [24, 33];

- improving the range and quality of referrals, thus increasing the likelihood that survivors will receive services such as forensic exams, counselling, EC, PEP and STI prophylaxis [5, 21, 22];

- sensitising the police and judiciary to improve attitudes towards abuse survivors [24, 34];

- developing legal tools that will increase women's personal and household security, such as divorce, division of marital property, child custody and child support [23, 32]; and

- ensuring the uniform and unbiased enforcement of existing sanctions [23, 24]. 


\section{Key Issues in SGBV trauma management}

Rape Trauma Syndrome [35] includes behaviour and personality changes that are manifested in a wide range of ways:

- physical manifestations include pain, nausea, vomiting, and headaches;

- behavioural manifestations may include eating disorders, sleep disturbances, abuse of drugs and alcohol and changes in normal day-to-day functioning.

Research suggests that life-long emotional trauma is often compounded by the prejudice and stigma associated with rape [18]. Qualitative studies indicate that women frequently consider emotionally abusive acts to be more devastating than physical violence [26].

Counselling is ideally conducted by an experienced general counsellor who has received specialised training in trauma counselling and HIV-testing in the context of sexual violence [12]. Privacy and confidentiality are central to reassuring survivors and securing their long-term safety. Both immediate trauma management and long-term counselling are necessary components of survivor care. There are several categories of counselling:

- Trauma counselling for crisis prevention aims to reduce immediate rape trauma disorder and long-term post-traumatic stress disorder, and needs to be prioritised for all patients, regardless of their time of presentation.

- HIV pre and post test counselling is recommended for all patients before he or she is tested for HIV, even if this requires that EC and PEP be administered prior to the HIV test.

- PEP adherence counselling has been shown to be effective in increasing adherence to PEP, and is recommended to coincide with PEP clinic follow-ups [6, 12].

- Counselling to prepare survivors for the justice system, while enabling access to legal counsel and aid, increases the likelihood that a survivor will complete the legal process [19]. The need for counselling is not necessarily limited to the survivor: the family and/or partners also undergo trauma and may require support [20].

There are special cases that require a modified counselling approach:

- Male survivors of abuse experience many of the same physical and psychological trauma symptoms as women. However, they are likely to have additional concerns about their masculinity and sexuality. The relative rarity of male survivors presenting for services may also contribute to stigma and feelings of powerlessness [16,26].

- The evaluation and counselling of children requires especial sensitivity and is of crucial importance in minimising the long-term physical and psychological consequences of sexual abuse. The carers or parents of abused children may need to be offered support and counselling. They need to give consent for the child's HIV test and to be prepared for a possible positive result $[16,12]$. 


\section{Literature references}

1. IGWG of USAID. 2006. Addressing Gender-Based Violence through USAID's Health Programs: A Guide for Health Sector Program Officers.Washington, DC.

2. Krug, Etienne, Linda Dalhberg, James Mercy, Anthony Zwi, and Rafael Lozano, Eds. 2002. World Report on Violence and Health. Geneva: WHO.

3. Andersson, N. et al. 2004. National cross-sectional study on views on sexual violence and risk of HIV infection and AIDS among South African school pupils. BMJ 2004: 329; 952-957.

4. Mugawe, D. \& A. Powell. 2006. Born to High Risk: Violence Against Girls in Africa. The African Child Policy Forum.

5. Bott, S., Morrison, A. and M. Ellsberg. 2005. Preventing and Responding to Gender-based Violence in Middle and Low-income Countries: A Global Review and Analysis. World Bank Working Paper Series 3618. Washington, DC: World Bank.

6. Askew, I. \& L. Ndhlovu. 2006. Developing a multisectoral and comprehensive response to Sexual and Gender Based Violence in East and Southern Africa. Project proposal to Swedish International Development Assistance from Population Council.

7. World Health Organization. 2001. Putting women first: Ethical and safety recommendations for research on domestic violence against women. Geneva: WHO.

8. Kim, J. C., Martin, L. J., \& Denny, L. 2003, "Rape and HIV post-exposure prophylaxis: addressing the dual epidemics in South Africa", Reprod. Health Matters., vol. 11, no. 22, pp. 101-112.

9. Stavropoulos, J. 2006. Violence Against Girls in Africa: A Retrospective Survey in Ethiopia, Kenya and Uganda. The African Child Policy Forum.

10. NASCOP. 2004a. Kenyan National ARV Guidelines. National AIDS and STD Control Programme, Ministry of Health, Nairobi.

11. NASCOP. 2004b. Kenyan National Clinical Manual for ARV providers. National AIDS and STD Control Programme, Ministry of Health, Nairobi.

12. Kenya Ministry of Health/Division of Reproductive Health. 2004. National Guidelines on the Medical Management of Rape/Sexual Violence (1st edition). Nairobi, Kenya, Tonaz Agencies.

13. Kilonzo, N \& M. Taegtmeyer. 2005. Comprehensive Post-Rape Care Services in Resource-Poor Settings: Lessons learnt from Kenya. Liverpool School of Tropical Medicine. Nairobi, Kenya, Liverpool VCT Kenya. Policy Briefings for Health Sector Reform: No. 6, September 2005.

14. World Health Organization. 2004b. Medical eligibility criteria for contraceptive use (3rd edition). Geneva: WHO.

15. Speight, C.G. et al. 2006. Piloting post-exposure prophylaxis in Kenya raises specific concerns for the management of childhood rape. Transactions of the Royal Society of Tropical Medicine and Hygiene. 100, 14-18.

16. World Health Organization. 2003. Guidelines for Medico-legal Care for Sexual Violence Victims. Geneva: WHO.

17. Malawi, Kenya and South Africa protocols.

18. Rose D, 1986, "Worse than Death: Psychodynamics of Rape Victims and the Need for Psychotherapy", The American Journal of Psychotherapy, 143:7

19. Policy Issues and a Policy Implementation Framework for Integrating Sexual Violence Services into the Reproductive Health Policy, a background document prepared for the Gender and Reproductive Health Rights Working Group in 2005 by Population Council and LVCT.

20. Kilonzo, N. 2003. Conceptualising Vulnerability to Sexual Violence \& HIV: Implications for Practical Responses. LVCT \& CARE.

21. Betron, M. \& E. Doggett. 2006. Linking Gender-Based Violence Research to Practice in East, Central and Southern Africa: A Review of Risk Factors and Promising Interventions. USAID/POLICY.

22. Global AIDS Alliance (GAA). 2006. Zero Tolerance: Stop the Violence Against Women and Children, Stop HIVIAIDS. Washington, DC: Global AIDS Alliance.

23. World Bank. 2006c. Addressing Violence against Women in Middle and Low-Income Countries: A Multi Sectoral Approach. Sector Operational Guide for the World Bank Gender and Development Group.

24. Bott, Sarah, Alessandra Guedes, Marla Cecilia Claramunt, Ana G, ezmes. 2004. Improving the Health Sector Response to Gender-based Violence: A Resource Manual for Health Care Managers in Developing Countries. New York: IPPF, Western Hemisphere Region (IPPF/WHR).

25. Saltzman, L., Fanslow, J., McMahon, P. and G. Shelley. 1999. Intimate partner violence surveillance: uniform definitions and recommended data elements. National Centre for Injury Prevention and Control.

26. World Health Organization. 2005. WHO Multi-country Study on Women's Health and Domestic Violence against Women: summary report of initial results on prevalence, health outcomes and women's responses. Geneva, World Health Organization.

27. Kishor, S. \& K. Johnson. 2004. Profiling Domestic Violence - A Multi-Country Study. Calverton, Maryland: ORC Macro.

28. Zambia Demographic and Health Survey. 2001-2002.

29. South Africa Demographic and Health Survey. 1998.

30. Kenya Demographic and Health Survey. 2003.

31. Ethiopia Demographic and Health Survey. 2005.

32. Guedes, A. et al. 2002. "Gender-based violence, Human Rights and the Health Sector: Lessons from Latin America". Health and Human Rights. Vol. 6 (1): 177-194.

33. United Nations General Assembly (UN-GA). 2006. In depth study on all forms of violence against women: Report of the Secretary-General.

34. Keesbury, J., Skibiak, J. \& M. Zama. 2006. Reducing unwanted pregnancy among victims of sexual assault: New windows of opportunity for Emergency Contraception. Draft paper: Population Council.

35. Burgess, A.W. \& L.L. Holmstrom. 1974. "Rape trauma syndrome", American Journal of Psychiatry, 1974, 131:981-986

36. Jejeebhoy, S. \& S. Bott. 2003. Non-consensual Sexual Experiences of Young People: A Review of the Evidence from Developing Countries. New Delhi: Population Council.

37. Kim, J. et al. 2007. "Developing an integrated model for post-rape care and HIV post-exposure prophylaxis in rural South Africa," FRONTIERS Final Report. Washington, DC : Population Council.

38. World Health Organization. 2005. Sexually Transmitted and Other Reproductive Tract Infections. Geneva: WHO 


\section{The African Regional SGBV Network}

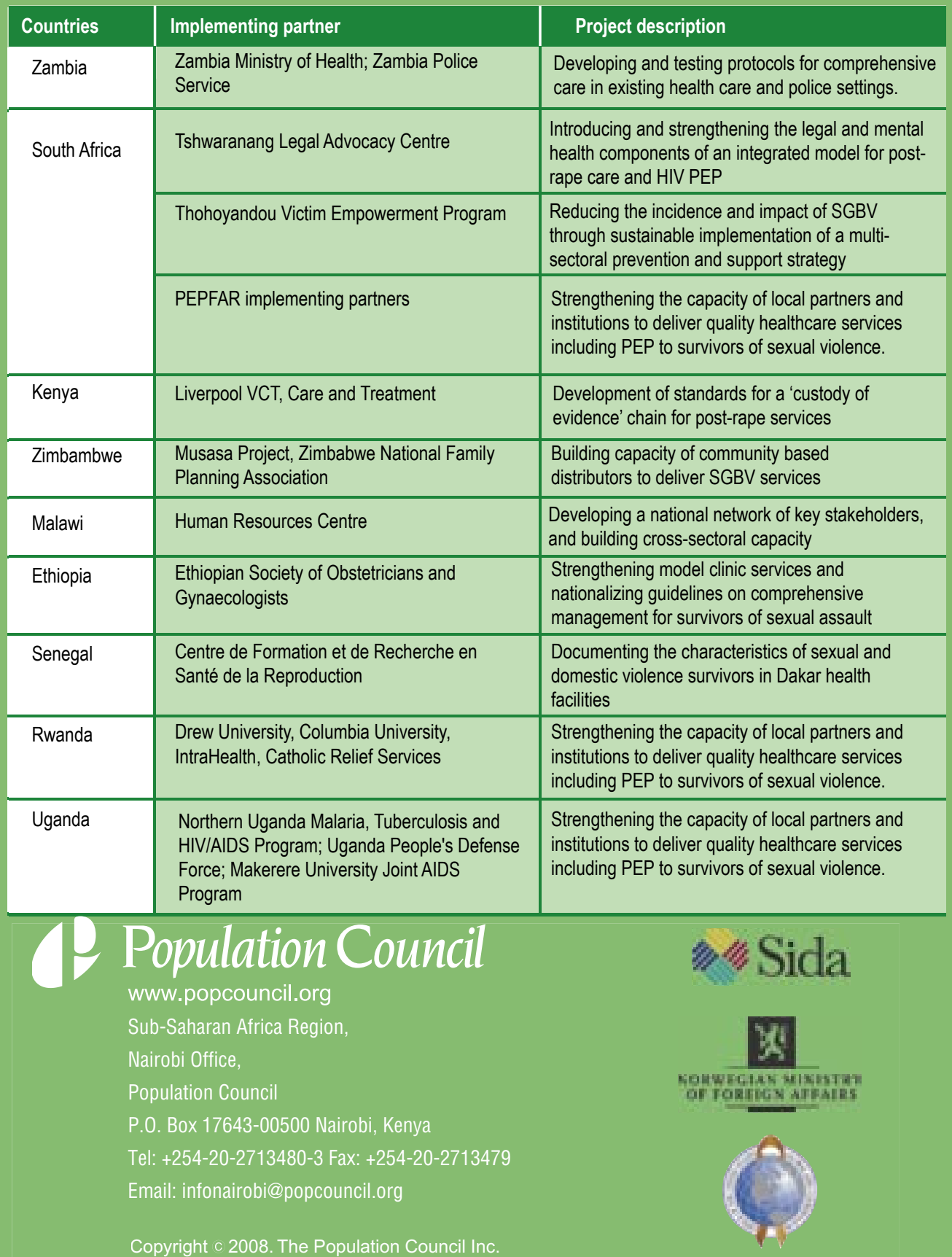

